\title{
Optical constants measurements of strongly absorbing media
}

\author{
Yutian Lu and Alfons Penzkofer
}

\begin{abstract}
An experimental arrangement is described for determination of refractive indices $n$ and extinction coeffi. cients $k$ of strongly absorbing liquids and solids over a wide spectral region. The reflectivity of parallel polarized light vs angle of incidence is measured, and the optical constants are calculated from the minimum parallel reflectivity and the corresponding Brewster angle. The accuracy of $n$ and $k$ determination is analyzed. The refractive indices and extinction coefficients of $0.4-\mathrm{M}$ rhodamine $6 \mathrm{G}$ in methanol are presented around the $S_{1}$ absorption band.
\end{abstract}

\section{Introduction}

The refractive indices of nonabsorbing liquids and solids are measured generally by prism refraction or total internal reflection with an accuracy of $\Delta n \simeq$ $10^{-4} \cdot 1^{1-3}$ For gases interference techniques are used $\left(\Delta n \simeq 10^{-7}\right)$. In weakly absorbing media $(k<0.03$, $\alpha$ $<7000 \mathrm{~cm}^{-1}$ ) the extinction coefficient $k$ is obtained from transmission measurements (accuracy $\Delta k / k \simeq$ $10^{-3}$ ), and the refractive index $n$ may be calculated from reflection measurements of nearly normal incident light $\left(\Delta n / n \simeq 10^{-3}\right) .^{4-8}$ In cases of strongly absorbing media both optical constants $n$ and $k$ have to be deduced from reflection ${ }^{9-12}$ or ellipsometric methods (comparison of reflected amplitude and phase with incident values). ${ }^{12-14}$ Extinction coefficients may be also determined by photoacoustic techniques. ${ }^{15-17}$

In this paper we describe an experimental arrangement for the measurement of $n$ and $k$ of strongly absorbing liquids and solids $(k \geqslant 0.02)$ over a wide spectral region. The reflectivity of parallel polarized light $R$ vs angle of incidence $\phi$ is measured simultaneously over a wide wavelength region. From the minimum reflectivity of parallel polarized light $R_{\|_{\min }}(\lambda)$, at the Brewster angle $\phi_{B}(\lambda)$, the optical constants $n(\lambda)$ and $k(\lambda)$ are calculated (method $F$ of Ref. 9 ). The accuracy of the system is analyzed. As an example the optical constants of a $0.4-\mathrm{M}$ solution of rhodamine $6 \mathrm{G}$ dissolved in methanol are determined in the wavelength region between 400 and $600 \mathrm{~nm}$.

The authors are with Universität Regensburg, Naturwissenschaftliche Fakultät II-Physik, 8400 Regensburg, Federal Republic of (iermany.

Rereived :25. June 1985.

(1)0:3-69:35/86/0220221-05\$02.00/0.

(c) 1985 Optical Society of America.

\section{Theory}

The reflection of light at the interface between two media is determined by the Fresnel laws. ${ }^{18-20}$ For parallel polarized light the ratio of reflected to incident electric field strength at an angle of incidence $\phi$ is 8

$$
\frac{E_{i 1}}{E_{i 1}}=\frac{n^{\prime 2} \mu \cos \phi-\left(n^{\prime 2}-\sin ^{2} \phi\right)^{1 / 2}}{n^{\prime 2} \mu \cos \phi+\left(n^{\prime 2}-\sin ^{2} \phi\right)^{1 / 2}} \text {. }
$$

where $n^{\prime}=n_{t}^{\prime} / n_{i}^{\prime}=\left(n_{t}-i k_{t}\right) / n_{i}^{\prime}=n-i k$ is the complex relative refractive index. $n_{i}^{\prime}=n_{i}-i k_{i}$ is the absolute refractive index of the incident medium and is real in the case of transparent media $\left(k_{i}=0\right)$. $n_{t}^{\prime}=$ $n_{t}-i k_{t}$ is the absolute complex refractive index of the medium under investigation. $n_{t}$ is the real refractive index, and $k_{t}=\alpha_{t} /(4 \pi \nu)$ is the extinction coefficient. $\alpha_{t}$ is the absorption coefficient at frequency $\nu=c \bar{\nu}$, where $\bar{\nu}$ is the wave number. $\mu=\mu_{i} / \mu_{t}$ describes the ratio of relative permeabilities. $\mu_{i}$ and $\mu_{t}$ are close to 1 for nonmagnetic media; so $\mu=1$ is used in our analysis.

The parallel reflectivity $R_{\|}=\left|E_{r \|} / E_{i \|}\right|^{2}$ is derived from Eq. (1) and reads 8

$R_{\|}=\frac{\mu^{2}\left(C^{2}+D^{2}\right) \cos ^{2} \phi+A-2 \mu A^{1 / 2} \cos \phi(C \cos B+D \sin B)}{\mu^{2}\left(C^{2}+D^{2}\right) \cos ^{2} \phi+A+2 \mu A^{1 / 2} \cos \phi(C \cos B+D \sin B)}$,

with $A=\left[\left(n^{2}-k^{2}-\sin ^{2} \phi\right)^{2}+4 n^{2} k^{2}\right]^{1 / 2}, B=0.5$ $\arctan \left[2 n k /\left(n^{2}-k^{2}-\sin ^{2} \phi\right)\right], C=n^{2}-k^{2}=\epsilon^{\prime}$, and $D=$ $2 n k=\epsilon^{\prime \prime}$ [relative permittivity $\epsilon=\epsilon^{\prime}-i \epsilon^{\prime \prime}=(n-i k)^{2}=$ $\left.n^{2}-k^{2}-i 2 n k\right]$.

In Fig. 1 the $R_{\|}(\phi)$ dependence is illustrated for $n=$ 0.7 (dashed curves) and $n=1.428(=1 / 0.7$, solid curves). Various extinction coefficients are used as labeled on the graph. In case of $n<1$ and $k=0$ total internal reflection occurs for $\phi \geq \phi_{c}=\arcsin (n)\left(\phi_{r}\right.$ is the critical angle). When absorption is present $(k>0)$ and $n<1$, the totally reflected light for $\phi \geq \phi_{c}$ is attenuated. This attenuated total reflection is often applied to obtain absorption spectra"1 (single attenuated total reflectance techniques, 22 multiple total reflectance techniques, ${ }^{23,24}$ internal reflection spectros- 


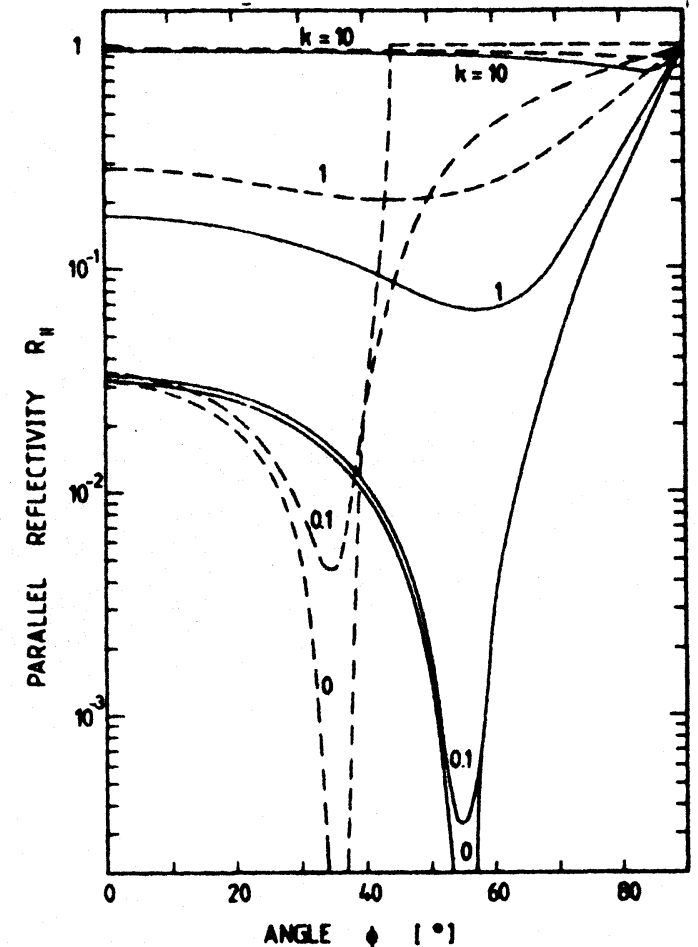

Fig. 1. Reflectivity curves for light polarized parallel to plane of incidence. Dashed curves, $n=0.7$; solid curves, $n=0.7^{-1}=1.428$.

copy ${ }^{25}$ ). For all refractive indices the Brewster angle $\phi_{\mathrm{B}}$ (angle of minimum parallel reflectivity) shifts from $\Phi_{\mathrm{B}}=\arctan (n)$ at $k=0$ to $\phi_{\mathrm{B}}=90^{\circ}$ at $k=\infty$. Up to $k \simeq$ $0.3, \phi_{\mathrm{B}}$ is practically independent of $k$, and the relation $n=\tan \phi_{\mathrm{B}}$ allows a simple determination of the refractive index. For $k>10$ the Brewster angle is $\phi_{\mathrm{B}}>8^{\circ}$, and the parallel reflectivity is $R_{\|}>0.7$. An accurate determination of $n$ and $k$ becomes difficult.

The location of $n$ and $k$ in a 2-D $\left(\phi_{\mathrm{B}}, R_{\|_{\min }}\right)$ plane is depicted in Fig. 2. This figure is a nomogram in that there exists a one-to-one correspondence between $\left(\phi_{\mathrm{B}}\right.$, $\left.\mathbf{R}_{\min }\right)$ pairs and $(n, k)$ pairs allowing the $n$ and $k$ determination by the $R_{\|}\left(\phi_{\mathrm{B}}\right)$ measurement. In the experimental arrangement described below $\phi_{\mathrm{B}}$ may be adjusted easily between 10 and $80^{\circ}$ spanning a refractiveindex region of $0.2 \leq n \leq 5$. The system allows reflectivity measurements down to $5 \times 10^{-6}$ so that extinction coefficients $0.02 \leqslant k \leqslant 5$ may be determined. For $n<1$ smaller $k$ values are measurable (metallic reflection behavior).

The accuracy of determination of $k$ and $n$ may be deduced from Figs. 3(a) and (b), respectively. In Fig. $3(a)$ the ratio of $(d k / k) /\left(d R_{\| \min } / R_{\| \min }\right)$ vs $k$ is plotted for various refractive indices. Over a wide range of $k$ $(0.01 \leq k \leq 1)$ and $n(1<n<100)$ the ratio is $(d k / k) /\left(d R_{\|_{\min }} / R_{\| \min }\right) \simeq 0.5$. The reflectivity may be determined with a relative error of $\Delta R_{\| \min } / R_{\| \min } \simeq$ \pm 0.01 resulting in a relative error of the extinction coefficient of $\Delta k / k \simeq \pm 0.005$. The ratio $\left(d n / d \phi_{\mathrm{B}}\right) / n$ vs $n$ is shown in Fig. $3(\mathrm{~b})$. It is independent of the extinction cuefficient $k$. The accuracy of $\phi_{\mathrm{B}}$ determination reduces for large $k$ values because the $R_{\|}(\phi)$ minima broaden with increasing $k$. For $k<0.1$ the Brewster

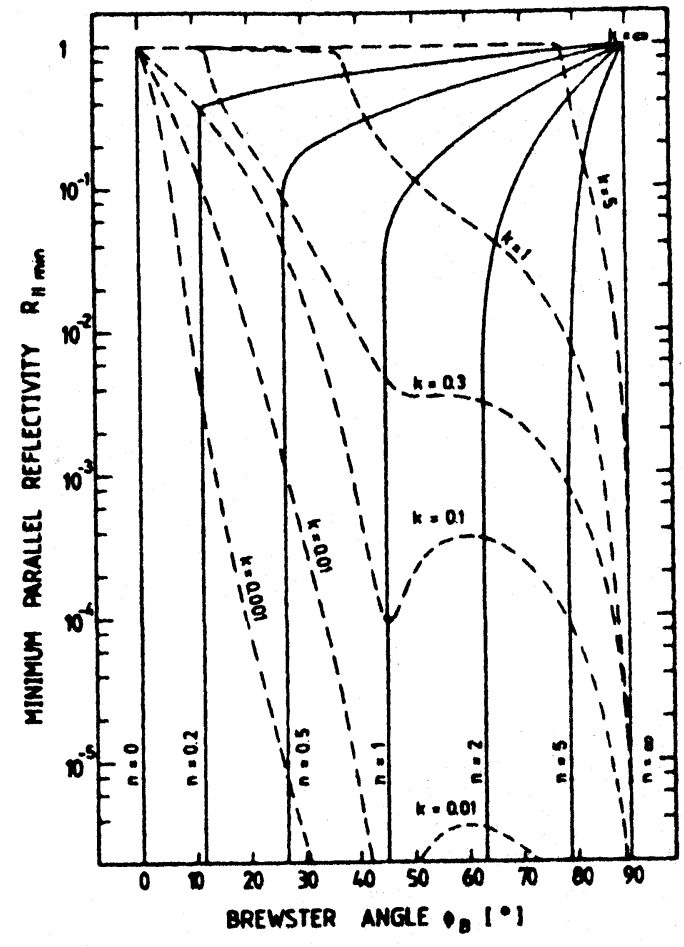

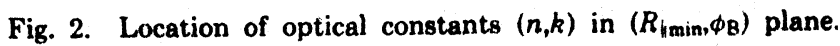
Dashed curves, $k=$ constant; solid curves, $n=$ constant.
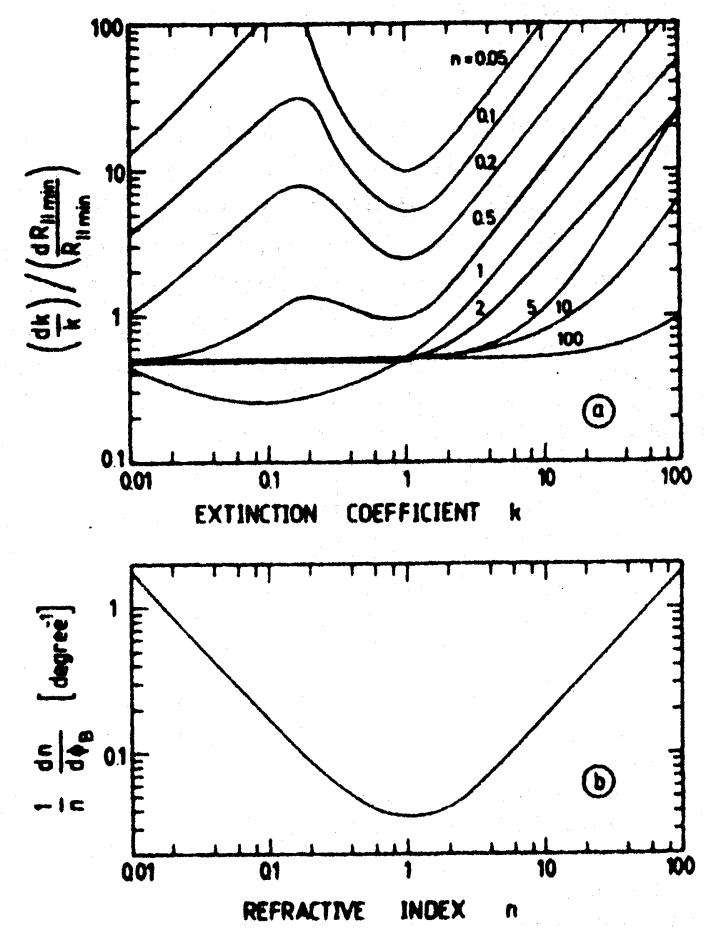

Fig. 3. Accuracy estimation of optical constants: (a) ration of $d k / k$ to $d R_{\|_{\min }} / R_{\| \min } \mathrm{vs}$ extinction coefficient $k$ for various refractiveindex values; (b) change of refractive index with Brewater angle $\left(d n / d \phi_{B}\right) / n$ vs refractive index $n$. The function is independent of extinction coefficient $k$. 
angle may be determined within $\Delta \phi_{\mathrm{B}} \simeq \pm 0.05^{\circ}$ giving relative errors between $\Delta n / n \simeq \pm 2 \times 10^{-3}$ at $n=1$ and $\Delta n / n \simeq \pm 0.01$ at $n=10$ or 0.1 . At $k=1$ the error in determination of the Brewster angle increases to $\Delta \phi_{\mathrm{B}} \simeq$ $\pm 1^{\circ}$ leading to $\Delta n / n \simeq \pm 0.04$ at $n=1$ and $\Delta n / n \simeq \pm 0.2$ at $n=10$ or 0.1 .

\section{Experiment}

The experimental arrangement is shown in Fig. 4. A 200 -W high pressure mercury lamp serves as the light source. A parallel light beam is formed by slit $S 1$, aperture $A 1$, and lens $L 1$. Lens $L 3$ focuses the beam to the sample $S$. Polarizers $P O 1$ and $P O 2$ transmit light polarized parallel to the plane of incidence of the sample. The angle of incidence is adjusted with mirror $M 1$ (plane aluminum mirror) on a precision rotation stage. Liquid samples are contained in open beakers inside a closed hollow prism with fused silica windows (see inset of Fig. 4, air-liquid interface with $n_{i}=1.00027$ ). The enclosure of the liquids hinders evaporation. The light beam reflected from the sample is directed to the spectrometer by mirror $M 2$. Lens $L 4$ focuses the beam to the input slit of a $30-\mathrm{cm}$ grating spectrometer $(600$ lines $/ \mathrm{mm}$ ). The light spectrum $S(\lambda)$ is registered by a silicon diode array detector DA (DARSS system of Tracor). The diode array system is connected to a microcomputer for data aquisition.

The light path from lens $L 3$ to mirror $M 1$ is made parallel to the sample surface by tilting mirror $M 1$ out of the light path entering a pentaprism above sample $S$ and retroreflecting the beam from the sample to lens $L 1$. The beam splitter $B S 2$, the aperture $A 2$, and a cross mark at beam stop $S T$ serve for reproducible alignment of the reflected beam. To get rid of fluctuations of the light source, the spectrum $S(\lambda)$ is normalized to part of the input light which is separated by beam splitter $B S 1$, focused into a fiber-optic light guide $L G$, and directed to a side region of the diode array detector (signal $S_{F}$ ).

The spectral signal $S(\lambda)$ of the substance under investigation is compared with the spectral signal $S_{\text {ref }}(\lambda)$ of a reference medium of known reflectivity $R_{\text {ref }}(\phi)$. For angles of incidence $\phi>42^{\circ}$ a total reflecting $60^{\circ}$ glass prism is used at the sample position. In case of $\phi$ $<42^{\circ}$ (no total reflection) a glass plate of known refractive-index dispersion is applied. The parallel reflectivity of the investigated substance is

$$
R_{i j}(\lambda, \phi)=R_{\text {Iref }}\left(\lambda, \phi^{\prime}\right) \frac{S(\lambda, \phi) / S_{F}}{S_{\text {ref }}\left(\lambda, \phi^{\prime}\right) / S_{F, \text { ref }}},
$$

where $\phi^{\prime}$ is an angle within the investigated region of $\phi$.

The experimental setup is very similar to the arrangement used for refractive-index measurement of moderately absorbing media ${ }^{8}$ and may be adopted to these measurements. Only the two polarizers have to be removed, and the sample region has to be changed for nearly normal incidence.

In the experiments the interested wavelength region is selected and split into discrete intervals (full spectral width $\lambda_{\min } \leq \lambda_{i} \leq \lambda_{\max }$; the spectral width of each discrete region is $\left.\Delta \lambda=\lambda_{i}-\lambda_{i-1}=3 \mathrm{~nm}\right)$. The reflec-

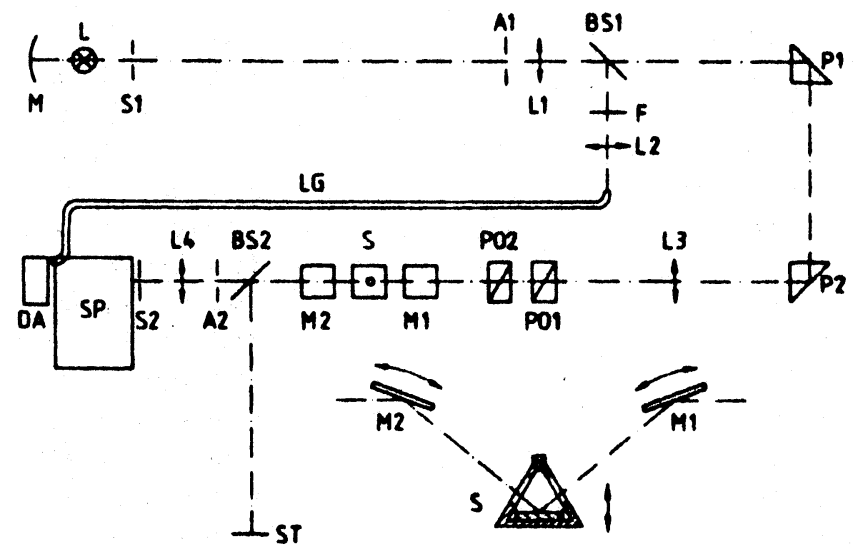

Fig.4. Experimental setup: $L$, lamp; $M$, light collecting mirror: $S_{1}$. $S_{2}$, slits (widths $S 1: 0.2 \mathrm{~mm}, S 2: 0.5 \mathrm{~mm}$ ): $A 1, A 2$, variable apertures. $L 1-L 4$, lenses (focal lengths $L 1: 1.33 \mathrm{~m}, L 2: 15 \mathrm{~cm}, L 3: 1 \mathrm{~m}$. $L 4: \quad 5 \mathrm{~cm}) ; B S 1, B S 2$, beam splitters; $F$, filters; $L G$. fiber-opric lightguide; $P 1, P 2,90^{\circ}$ deflection prisms; $P O 1, P() 2$, Glan polarizers: $M 1, M 2$, plane aluminum mirrors; $S$, sample; $S T$, beam stop with cross hair; SP, 30-cm spectrometer (grating with 600 lines $/ \mathrm{mm}$ ): D.A. silicon diode array detection system (Tracor DARSS) connected to the microcomputer. The inset shows the sample region in more detail.

tivity $R_{\|}(\lambda)$ is measured for a discrete set of angles $\phi_{\text {, }}$ $\left(\phi_{\min } \leq \phi_{j} \leq \phi_{\max }, \Delta \phi=\phi_{j}-\phi_{j-1}=1^{\circ}\right)$ around the region of the expected Brewster angles $\phi_{B}(\lambda)$. The matrix of data $R_{\|}\left(\lambda_{i}, \phi_{j}\right)$ is analyzed numerically. For each wavelength $\lambda_{i}$ the minimum parallel reflectivity $R_{\|, \min }\left(\lambda_{i}\right)$ at angle $\phi_{\mathrm{B}}\left(\lambda_{i}\right)$ is determined. Spline interpolation is used to find $\phi_{\mathrm{B}}$ and $R_{\|, \min }$ within the measured grid. The resulting set of data $R_{\|, \min }\left(\lambda_{i}\right)$ and $\phi_{\mathrm{B}}\left(\lambda_{i}\right)$ is used to calculate $n\left(\lambda_{i}\right)$ and $k\left(\lambda_{i}\right)$ exploiting Eq. (2). [Initial values of $n$ and $k$ are taken from Fig. 2. $k$ is iteratively adjusted to fit $R_{\|_{\min } .} R_{\|}\left(\phi_{\mathrm{B}} \pm \Delta \phi, n, k\right)$ is calculated to check the minimum condition. If it fails the procedure is repeated with improved $n$ until $n$ and $k$ give $R_{\| \min }$ and $\phi_{\mathrm{B}}$.]

In a test a BK7 glass plate at $\lambda=546.1 \mathrm{~nm}$ was studied. The data were $\phi_{\mathrm{B}}=56.65^{\circ}$ and $R_{\|, \min }=2 \times$ $10^{-6}$ corresponding to $n=1.52$ and $k=0.008$. The true refractive index is $=1.51872$ (Schott data sheet) indicating an experimental error of $\Delta n / n=0.001$. The minimum reflectivity of the transparent medium should be zero. The remaining parallel reflectivity is due to imperfect parallel polarization, light depolarization, and light scattering. The remaining parallel reflectivity sets a lower limit of $\sim 0.01$ for the $k$ determination.

\section{Results}

The described system was used to measure the optical constants of $0.4-\mathrm{M}$ rhodamine $6 \mathrm{G}$ dissolved in methanol within a wavelength region from 400 to 600 $\mathrm{nm}\left(S_{0}-S_{1}\right.$ absorption peak at $\left.530 \mathrm{~nm}\right)$. Figure 5(a) depicts the measured $n$ and $k$ values. $n$ is obtained over the full wavelength region by the applied tech. nique. The $k$ values obtained by the $R_{\|}\left(\phi_{\mathrm{B}}\right)$ measurement are restricted to $k>0.02$ because of background signal. The smaller $k$ values were determined by transmission measurements in $10-\mu \mathrm{m}$ cells. 


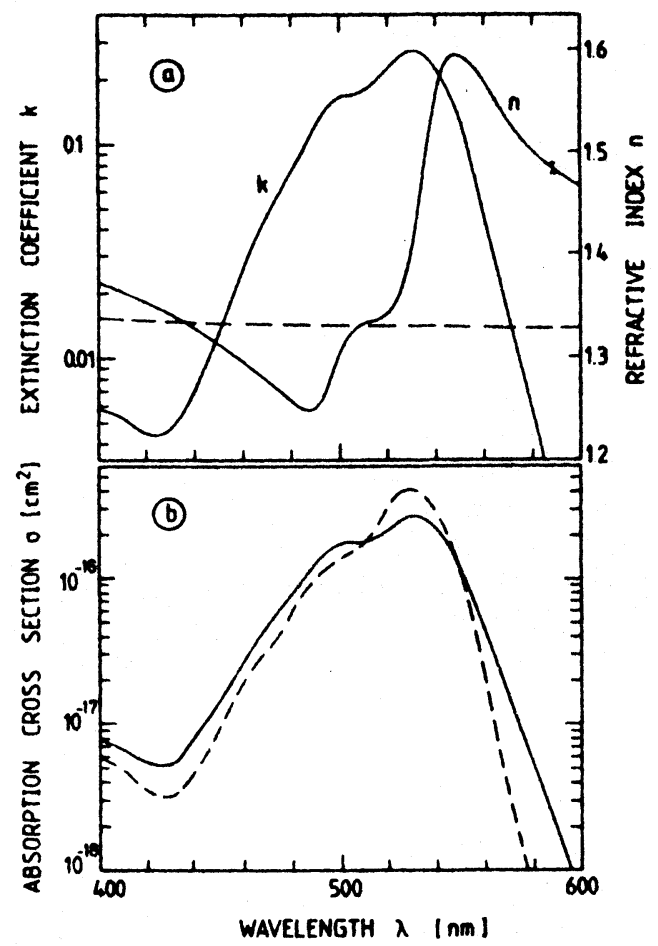

Fig. 5. (a) Refractive indices and extinction coefficients vs wavelength for $0.4-\mathrm{mol} /$ liter rhodamine $6 \mathrm{G}$ in methanol. Temperature $21^{\circ} \mathrm{C}$. The dashed curve represents the refractive index of the solvent methanol. (b) Absorption cross section vs wavelength for rhodamine $6 \mathrm{G}$ in methanol. Dashed curve, concentration $10^{-5} \mathrm{~mol}$ /liter; solid curve, concentration $0.4 \mathrm{~mol} /$ /iter.

From the extinction coefficient $k$, the absorption cross section $\sigma$ of the investigated dye is calculated by the relation $\sigma=\alpha_{t} / N=4 \pi \tilde{\nu}_{i} k / N$, where $N=N_{A} C$ is the number density of dye molecules. $\left(N_{A}=6.022045 \times\right.$ $10^{23} \mathrm{~mol}^{-1}$ is the Avogadro constant, $C$ is the concentration.) The solid curve in Fig. 5(b) represents the obtained absorption cross sections for 0.4-M rhodamine $6 \mathrm{G}$ in methanol, while the dashed curve belongs to a rhodamine $6 \mathrm{G}$ concentration of $10^{-5} \mathrm{~mol} /$ liter. The $0.4-\mathrm{mol} / \mathrm{liter}$ curve is flatter than the $10^{-5}-\mathrm{mol}$ hiter curve. ${ }^{26,27}$ The difference in the absorption cross-section spectrum is thought to be due to the mutual interaction of rhodamine $6 \mathrm{G}$ molecules at high concentration. It should be noted that at a concentration of $0.4 \mathrm{~mol} /$ iter (number density is $N=2.4 \times 10^{20}$ $\mathrm{cm}^{-3}$ ) the average distance between the two dye molecules is $1.6 \mathrm{~nm}$, while the molecular dimension of a rhodamine $6 \mathrm{G}$ molecule is $\sim 1.1 \times 1.1 \times 0.4 \mathrm{~nm}^{3}$.

\section{Conclusions}

The described system determines the extinction coefficient $k$ and refractive index $n$ by measuring the minimum reflectivity of parallel polarized light and the corresponding angle of incidence (Brewster angle). The technique allows one to measure the optical constants $n$ and $k$ of strongly absorbing media $(k>0.02)$. The technique was used to measure the absorption spectrum of a highly concentrated dye to get information on any deviations from Beer's law.
The authors are grateful to T. Ascherl for his technical assistance. They thank the Rechenzentrum of the University for computer time.

Yutian $\mathrm{Lu}$ is on leave from the Institute of Optics and Fine Mechanics, Academia Sinica, Shanghai, China.

\section{References}

1. H. Kessler, "Optische Konstanten," in Handbuch der Physik, Vol. 18: Geometrische Optik, optische Konstante, optische Instrumente, H. Geiger and K. Scheel, Eds. (Springer-Verlag. Berlin, 1927), p. 623.

2. G. E. Fishter, "Refractometry," in Applied Optics and Optical Engineering, Vol.5, P'art 1, R. Kingslake, Ed. (Academic, New York, 1967), p. 363.

3. K. Schmid and A. Penzkofer, "Refractive-Index Measurements with a Pellin-Broca Prism Apparatus," Appl. Opt. 22, 1824 (1983).

4. L. G. Schulz and F. R. Tangherlini, "Optical Constants of Silver. Gold, Copper, and Aluminum. II. The Index of Refraction n," J. Opt. Soc. Am 44, 362 (1954).

5. F. Oswald and R. Schade. "Über die Bestimmung der Optixhen Konslanten von Hulbleitern des Typus $A^{{ }^{I I I}}-B^{v}$ im Infraroten." Z. Naturforsch. Teil A 9,611 (1954).

6. L. I. Alperovich, "Use of Layers of Uneven Thickness for Measuring Optical Constants of Liquids in the Region of Strong Absorption," Opt. Spectrosc. 15, 181 (1963).

7. E. Denton, R. D. Campbell, and S. G. Tomlin, "The Ietermination of the Optical Constants of Thin Films from Measureinents of Reflectance and Transmittance at Normal Incidence," $J$. Phys. D 5, 852 (1972). 
8. W. Leupacher and A. Penzkofer, "Refractive-Index Measurement of Absorbing Condensed Media," Appl. Opt. 23, 1554 (1984).

9. S. P. F. Humphreys-Owen, "Comparison of Reflection Methods for Measuring Optical Constants without Polarimetric Analysis, and Proposal for New Methods Based on the Brewster Angle," Proc. Phys. Soc. London 77, 949 (1961).

10. I. Simon. "Spectroscopy in Infrared by Reflection and Its Use for Highly Absorbing Substances," J. Opt. Soc. Am. 41, 336 (1951).

11. M. R. Philpott, "Optial Reflection Spectroscopy of Organic Solids." Ann. Rev. Phys. Chem. 31, 97 (1980).

12. L. K. ('hopra, Thin Film Phenomena (McGraw-Hill, New York, 1969), Chap. 11.

13. J. R. Beattie and G. K. T. Conn, "Optical Constants of Metals in the Infra-Red-Principles of Measurement," Philos. Mag. 46, $222(1955)$

14. R. M. A. Azzam, "Explicit Determination of the Complex Refractive Index of an Absorbing Medium from Reflectance Measurements at and near Normal Incidence," J. Opt. Soc. Am. 72 1439 (1982).

15. R. Rosencwaig, Photoacoustics and Photoacoustic Spectroscopy (Wiley, New York, 1980).

16. J. F. McChelland, "Photoacoustic Spectroscopy," Anal. Chem. 55, 89A (1983).

17. J. B. Kinney and R. H. Staley, "Applications of Photoacoustic Spectroscopy," Ann. Rev. Mater. Sci. 12, 295 (1982).

18. W. König, "Electromagnetische Lichttheone," in Handbuch der Physik, Vol. 20: Licht als Wellenbewegung, H. Geiger and K. Scheel, Eds. (Springer-Verlag, Berlin, 1928), p. 242.

19. H. Gobrecht, "Dispersion und Absorption des Lichtes," in Bergmann-Schaefer, Lehrbuch der Experimentalphysik, Band III: Optik. H. Gobrecht, Ed. (Walter de Gruyter, Berlin, 1974), p. 265.

20. R. W. Ditchburn, Light (Academic, London, 1976).

21. W. W. Wendlandt and H. C. Hecht, Reflectance Spectroscopy (Interscience, New York, 1966).

22. J. Fahrenfort, "Attenuated Total Reflection. A New Principle for the Production of Useful Infra-Red Reflection Spectra of Organic Compounds," Spectrochim. Acta 17, 698 (1961).

23. N. J. Harrick, "Study of Physics and Chemistry of Surfaces from Frustrated Total Internal Reflections," Phys. Rev. Lett. 4, 224 (1960)).

24. J. Fahrenfort and W. M. Visser, "On the Determination of Optical Constants in the Infrared by Attenuated Total Reflection," Spectrochim. Acta 18, 1103 (1962).

25. N. J. Harrick. Internal Reflection Spectroscopy (Interscience, New York, 1967).

26. E. G. Baranova. "Study of the Association of Rhodamine 3G in Ethanol and Glycerol Solutions," Opt. Spectrosc. 13, 452 (1962).

27. .. E. Selwyn and J. L. Steinfeld, "Aggregation Equilibria of Xanthene Dyes," J. Phys. Chem. 76, 762 (1972).

Books contunued from paxe 2013

Optical Holography. By P. HARIHARAN. Cambridge University Press, Cambridge, 1984.319 pp. $\$ 69.50$.

For nearly 15 years the bible in holography has been OPTICAL HOL.)(iRAPHY by Collier, Burckhardt, and Lin. During these 15 years there have been many developments in holography; a book of the breadth and quality of Hariharan's is long overdue.

In the P'reface Hariharan states that his "aim in writing the book is to present a self-contained treatment of the principles. techniques, and applications of optical holography, with particular emphasis on reccnt developments." While this book covers both new and old material in suificient detail to be self-contained, it does not go into excessive detail. Instead it is generous in giving references to the original papers. Because of this, we believe that the author has achieved his goal.

The book contains fifteen chapters, four appendices, and a list of some 700 references. These fifteen chapters are split into two sections. The first seven chapters concentrate on the basics of holography, recording media, and the making of holograms. Chapter 1 provides a short historical overview of holography. Chapters 2-4 outline the basic wavefront reconstruction process, describe the reconstructed image and its aberrations, and discuss the types of hologram. The next three chapters deal with light sources and the recording media. Chapter 5 describes optical systems and light sources for making holograms. Characteristics of recording media are presented in Chap. 6, and Chap. 7 lists some practical recording media.

The second half of the book deals with various applications of holography such as displays, color holography, computer-generated holograms, and holographic interferometry. Chapters 8 and 9 de scribe holograms for displays and color holography. These chapters are of particular interest because they cover a lot of excellent materi. al that has not been dealt with adequately in previous holography books. Chapter 10 discusses the theory and applications of computer-generated holograms. Special holographic techniques such as polarization recording, incoherent holography, and the copying of holograms are covered in Chap. 11. The next two chapters discuss applications in imaging such as particle sizing, correction of aberrated wave fronts, high-resolution projection imaging, evanescent-wave holography, holographic diffraction gratings and optical elements, and information storage and processing.

Last, there are two chapters on the largest use of holography: holographic interferometry. Chapter 14 contains an up-to-date description of heterodyne-holographic interferometry as well as traditional techniques for holographic nondestructive testing. Then Chap. 15 gives an excellent discussion of the measurement of vibrations, photoelasticity, and contouring techniques. Finally, the book is rounded out with appendices containing short descriptions of Fourier transforms, wave propagation and diffraction, interference and coherence, speckle, and the H\&D curve.

Hariharan has done something that we thought was impossible. He has succeeded in writing a book on general holography that we like better than all previous books. We strongly recommend it.

JAMES C. WYANT

\section{KATHERINE CREATH}

IEEE ElectroTechnology Review 1984. Edited by RICHARD M. WHITE. Institute of Electrical \& Electronic Engineers, NY, 1985. 92 pp. $\$ 5.00$.

This first edition of ElectroTechnology Review summarizes new developements in the fields that comprise electrotechnology for readers who have some technical know-how. This volume-inaugurating what is hoped to be an annual review series-contains 32 short reviews of areas of electrical and electronic engineering and computers. There are three two-page surveys of special interest under the heading Optical Materials. Devices and Applications. I. P. Ippen has an account of the shortening of ultrashort uptical pulses in which the pulse time has been reduced since 1965 from 10 ps $\left(10^{-12}\right)$ to 16 fs $\left(1.6 \times 10^{-14}\right)$ in 1984 by using the colliding-pulsemode-locking (CPM) principle. Reliable pulses shorter than $100 \mathrm{fs}$ can be produced with a CPM dye laser using the interaction of two oppositely directed pulses in a thin saturable absorber. Shyh Wang describes tunable semiconductor lasers which employ various interferometric principles to control the laser wavelength. Jiseph $T$ Longo shows how improved IR detector arrays can be made using stable crystalline layers of mercury-cadmium-telluride krown epitaxially on single-crystal sapphire instead of bulk CdTe.

FRANKLIN S. HAKKIS. JR. continued un pare 275 